\title{
TRASTORNOS DEL COMPORTAMIENTO EN DAÑO CEREBRAL ADQUIRIDO
}

\section{BEHAVIOURAL DISORDERS AFTER ACQUIRED BRAIN INJURY}

\author{
Ignacio SÁnchez-Cubillo ${ }^{1,2}$, Nerea LeRTXundi ${ }^{3}$, Jose IGNACIO QuemadA $^{1,4}$, \\ RAQUEL RUIZ-RUIZ ${ }^{5}$ \\ Ignacio Sánchez-Cubillo: Instituto de Investigaciones Psiquiátricas. C/ Egaña, 10; 48010 Bilbao. \\ e-mail: sanchez-cubillo@gmx.net \\ ${ }^{1}$ Instituto de Investigaciones Psiquiátricas, Bilbao. \\ ${ }^{2}$ Facultad de Psicología. Universitat des Illes Balears. Palma de Mallorca. \\ ${ }^{3}$ Centro de Día de Daño Cerebral Aita Menni. San Sebastián. \\ ${ }^{4}$ Unidad de Daño Cerebral. Hospital Aita Menni. Bilbao. \\ ${ }^{5}$ Clinica Ubarmin. Pamplona.
}

\section{Resumen}

Las alteraciones de conducta como consecuencia de un daño cerebral adquirido, son consideradas como algunos de los déficit más relevantes en la neuropsicología. Su importancia se pone de manifiesto en tres aspectos fundamentales de la neuropsicología clínica: conceptualización del déficit, evaluación y rehabilitación. (1) Conceptualmente, se trata de un grupo confuso y precariamente definido de síntomas, que tradicionalmente se han englobado bajo el epígrafe "síndrome del lóbulo frontal (SLF)». Su fraccionamiento en subtipos ha permitido una aproximación a estos déficit más exhaustiva y específica, aunque todavía no es posible alcanzar niveles explicativos con evidencias sólidas. Recientes propuestas teóricas (marcadores somáticos, Teoría de la Mente, etc.) aportan marcos atractivos desde los que comenzar la búsqueda de causas. (2) Por otra parte, en el proceso evaluador existe una manifiesta falta de instrumentos estandarizados para su descripción individualizada. Este hecho es especialmente llamativo si se compara con la amplia oferta de test y escalas existentes para otros déficit (memoria, atención, etc.). (3) Por último, el proceso rehabilitador se ve interferido en gran medida por la presencia de un trastorno de este tipo, lo cual remarca su relevancia. Además, estas patologías son especialmente persistentes, pudiendo incluso empeorar a lo largo del tiempo, si no existe una intervención específica. Enfoques como la terapia de conducta y la psicofarmacología han demostrado ser útiles en la reducción de síntomas no deseados, aunque en algunos casos, como en la apatía, el tratamiento apenas resulta eficaz.

\section{Palabras Clave}

Alteraciones de conducta, desinhibición, apatía, trastorno orgánico de personalidad, neuropsicología.

\begin{abstract}
Behavioural disorders (BD) after brain injury are some of the most relevant issues in neuropsychology. This relevance is remarkable in three main areas of clinical neuropsychology: conceptual issues, assessment and rehabilitation. (1) BD is an heterogenous group of superficially defined symptoms, traditionally included under the term Frontal Lobe Syndrome. Its fractionning into subtypes allowed a more comprehensive and specific approach to $\mathrm{BD}$, although evidence is still not able to explain its causes. Recent theoretical approaches (somatic markers, Theory of mind) suggest interesting directions for research in this area. (2) Assessment of BD is not as developed as other areas (memory, attention...), and it lacks of standarized tests with satisfying psychometric requirements. (3) Rehabilitation processes are severily interfered by $\mathrm{BD}$, which are generally chronic and may even get worse with the years. Behaviour Therapy and psychopharmacological interventions have proven some usefullness in symptom reduction, although in symptoms of diminished motivation (apathy) treatment often fails to succeed.
\end{abstract}

\section{Key Words}

Behavioural disorders, Disinhibition, Apathy, Organic Personality Disorders, Neuropsychology. 


\section{Introducción}

Las alteraciones de conducta (equivalentes a los trastornos orgánicos de la personalidad), como consecuencia de un daño cerebral adquirido (DCA), son consideradas como algunas de las más relevantes en la neuropsicología. Su importancia se pone de manifiesto en tres aspectos fundamentales de la neuropsicología clínica: conceptualización del déficit, evaluación y rehabilitación.

Conceptualmente, se trata de un grupo confuso y precariamente definido de síntomas, que tradicionalmente se han englobado bajo el epígrafe «síndrome del lóbulo frontal (SLF)». En el proceso evaluador, existe una manifiesta falta de instrumentos estandarizados para su descripción individualizada. Este hecho es especialmente llamativo si se compara con la amplia oferta de test y escalas existentes para otros déficit (memoria, atención, etc.). El proceso rehabilitador, a todos los niveles, se ve interferido en gran medida por la presencia de un trastorno de este tipo. Además, estas patologías son especialmente persistentes, pudiendo incluso empeorar a lo largo del tiempo, si no existe una intervención específica.

\section{Aspectos conceptuales}

Para comprender cómo se entienden actualmente las alteraciones del comportamiento en DCA resulta conveniente considerar brevemente cuál ha sido el origen y evolución del concepto SLF (Benton, 1991). Tras la descripción clásica del paciente Phineas Gage por John M. Harlow a mediados del siglo XIX (Harlow, 1848, 1868), comenzaron a aparecer trabajos en los que se documentaban cambios en la personalidad y el humor de pacientes como consecuencia de lesiones del lóbulo frontal. En 1888, Moritz Jastrowitz utilizó el término «moria» para referirse a la agitación extraña y jovial de una serie de pacientes con tumores en el lóbulo frontal (Jastrowitz, 1888). En el mismo año, Leonore Welt describió la conducta maliciosa, agresiva, propensa a gastar bromas pesadas y a hacer chistes malos de un caso con traumatismo craneoencefálico (TCE) (Welt, 1888). Dos años después, Hermann Oppenheim presentó el caso de un paciente que presentaba una peculiar adicción a realizar bromas y chistes triviales, de naturaleza predominantemente sarcástica. "Witzelsucht» (literalmente, adicción a los chistes) fue el vocablo que acuñó para referirse esta conducta (Oppenheim, 1890). Estas descripciones clínicas supusieron las bases esenciales en la fundación del concepto SLF (Benton, 1991).

Paulatinamente fueron apareciendo descripciones clínicas similares, como las de Quensel, Schuster o Bernhardt (Bernhardt \& Borchardt, 1909; Quensel, 1914; Schuster, 1902), que añadieron rasgos clínicos como la impulsividad, la agresividad, el infantilismo y la conducta grosera y socialmente inapropiada. Tras la Primera Guerra Mundial, Feuchtwanger (Feuchtwanger, 1923) analizó los síntomas que presentaban los pacientes con lesiones prefrontales ${ }^{1}$, concluyendo que aparecían con mayor frecuencia que trastornos sensoriomotores, lingüísticos o de memoria, más habituales en lesiones no frontales. Además, este autor recalcó la importancia de la personalidad premórbida como influencia en la presentación del cuadro clínico, cuya variabilidad incluía depresión o apatía, euforia, psicopatía o histeria. Esta diversidad de síntomas le sirvió para considerar que un concepto simplista como el SLF no era sostenible (Benton, 1991).

A lo largo de las décadas posteriores, se fue ahondando en las descripciones clínicas de los pacientes con lesiones prefrontales. A pesar de que los influyentes trabajos de algunos autores (Hebb, 1945; Vincent, 1936) sugerían que estas lesiones no tenían efecto alguno (lo cual explica la visión del córtex prefrontal como «área silente del cerebro») se fue asentando la idea de que las lesiones de la corteza prefrontal (CPF) provocaban exacerbaciones de la personalidad premórbida, así como nuevas conductas patológi-

\footnotetext{
1 A partir de 1947 se empleó el término «corteza prefrontal» para referirse a las áreas del lóbulo frontal afectadas en este tipo de pacientes, aunque hasta entonces se utilizaba el término lóbulo frontal de forma general.
} 
cas tales como desinhibición, agresividad, irritabilidad, apatía, etc.

La situación actual del síndrome del lóbulo frontal es la de un término en creciente desuso, precisamente por la gran variabilidad de síntomas que ya señalara Feuchtwanger (Feuchtwanger, 1923). La tendencia actual es la de separar este concepto global en varios síndromes más específicos, desde la neuropsiquiatría, o bien a describir los procesos cognitivos subyacentes y su afectación, desde la neuropsicología cognitiva.

\section{a) La perspectiva neuropsiquiátrica}

Las clasificaciones diagnósticas internacionales (CIE, DSM) consideran estas alteraciones, y lo hacen bajo el epígrafe Trastorno orgánico de personalidad (CIE-10; F07.0) o Cambio de personalidad debido a enfermedad médica (DSM-IV). Además, incluyen varios subtipos (desinhibido, lábil, agresivo, apático, mixto, etc.) para reflejar la variabilidad de presentación del trastorno.

Como evolución desde el síndrome unitario hacia una descripción más pormenorizada de la sintomatología subsiguiente a las lesiones prefrontales, se propuso su división en tres grupos, en función de las áreas lesionadas y de las características conductuales (Duffy \& Campbell, 1994; Moreno Gea \& Blanco Sánchez, 1997):

1) Un síndrome desinhibido (también llamado pseudopsicopático), que se observa tras la lesión de las áreas orbitofrontales (o del circuito del que forman parte (Cummings, 1998)) del CPF, caracterizado por impulsividad, escasa empatía, afecto inapropiado e infantil, distractibilidad, egocentrismo, inestabilidad emocional, falta de juicio social, irritabilidad y dificultad para adecuar el comportamiento al entorno social.

2) Un síndrome apático, tras lesionarse áreas del cíngulo anterior (o de su circuito (Cummings, 1998)), que incluye apatía extrema, espontaneidad del comportamiento reducida, lenguaje empobrecido, acinesia e indiferencia al dolor.
3) Un síndrome disejecutivo, implicado en lesiones dorsolaterales del CPF (y del circuito correspondiente (Cummings, 1998)), que no suele incluirse en los trastornos de conducta, ya que se caracteriza por dificultades de planificación, abstracción, fluidez, ordenación temporal, en definitiva, funciones ejecutivas, aunque puede ir asociada cierta apatía y embotamiento afectivo.

La perspectiva sindrómica es útil desde el punto de vista clínico, ya que permite la comunicación ágil entre profesionales. Por otra parte, se ha criticado duramente desde las posiciones dimensionales de la neuropsiquiatría cognitiva, afirmando que las categorías no suelen reflejar la realidad de los pacientes. Se ha llegado a afirmar que las personas que reciben estos diagnósticos lo único que tienen en común es precisamente la categoría diagnóstica (Halligan \& David, 2001). Por ello, si se pretende ir más allá de la descripción y se asumen objetivos de tipo explicativo, resulta relevante analizar las aproximaciones cognitivas a los trastornos que nos ocupan.

\section{b) La perspectiva de la neuropsicología cognitiva:}

Tradicionalmente, la neuropsicología cognitiva ha centrado sus esfuerzos principalmente en procesos como la atención, la memoria, etc., pero ha mantenido durante mucho tiempo fuera de su foco de atención las alteraciones de conducta. Esto se debe, en parte, a su gran dificultad de estudio, pero también debido a que se ha considerado área de estudio de la psiquiatría. Afortunadamente, en los últimos años el interés por las alteraciones de conducta, especialmente las de tipo desinhibido y, en menor medida, la apatía, ha aumentado espectacularmente y su estudio produce gran cantidad de publicaciones científicas al más alto nivel.

\section{La desinhibición conductual:}

Esta patología equivale al síndrome desinhibido o pseudopsicopático descrito previamente. La utilización del término desinhibición está 
en desuso porque conduce a un equívoco: la idea de que existe un déficit en la capacidad de inhibir impulsos, deseos o instintos. En un trabajo reciente se analizaron los procesos inhibitorios (siguiendo la clasificación de Nigg de los mismos (Nigg, 2000)) y no se hallaron correlaciones entre la presencia de déficit en los mismos y la conducta desinhibida de los sujetos del grupo experimental (Sánchez-Cubillo, Quemada, Muñoz Céspedes, Furundarena, \& GarcíaValcarce, 2003). Por ello, los procesos que subyacen a esta patología podrían ser de otra naturaleza. En este sentido, existen varias propuestas teóricas para dar cuenta de los mecanismos alterados (Sánchez-Cubillo, Muñoz-Céspedes, \& Quemada, 2004).

Una de las más populares se encuentra en los trabajos del equipo de Antonio Damasio. Sus trabajos se centran en explicar por qué estos pacientes toman decisiones a lo largo de su vida de un modo persistentemente erróneo y perjudicial para los propios intereses. Para ello, formularon la hipótesis del marcador somático (Damasio, 1996), según la cual, la corteza ventromedial (u orbitofrontal) asociaría situaciones concretas (inversiones arriesgadas, accidentes por realizar actividades peligrosas, conductas inadecuadas en el trabajo, etc.), sus consecuencias (pérdidas económicas, daño físico, pérdida del empleo, etc.) y la emoción (primaria) que provocan dichas consecuencias (ansiedad, miedo, etc.). De esta forma, la corteza ventromedial "avisaría» al sujeto, en una situación subsiguiente (una nueva inversión) de que existe un peligro (pérdida económica) y lo hace mediante la creación de estados emocionales similares a los sentidos anteriormente (ansiedad, miedo). Así, el sujeto anticiparía las consecuencias de sus actos en forma de emoción corporal (sensación de ansiedad ante la posibilidad de invertir y perder dinero). La lesión orbitofrontal provocaría la pérdida de esta capacidad, con lo que el paciente no podría anticipar las consecuencias de sus actos, al menos no desde un punto de vista emocional, y tomaría decisiones de forma desventajosa.

Desde otra perspectiva, parece prometedora la aplicación a estos pacientes del estudio de la cognición social, o capacidad de entender y razonar acerca del entorno social. Paulatinamente comienza a describirse una red neural responsable de la cognición social, que incluye varios mecanismos (Singer et al., 2004). Probablemente el más conocido sea el denominado «Teoría de la mente» (ToM) o mentalización. Pero existen otros mecanismos implicados, sin los cuales es difícil adaptar la conducta al entorno social, como p. ej. el reconocimiento de expresiones faciales y la imitación motora.

La ToM sugiere que los seres humanos, así como los primates superiores, poseemos la capacidad de establecer una teoría sobre cómo funciona la mente del otro. Es decir, tenemos unas premisas que nos ayudan a entender por qué el otro se comporta de un determinado modo, en qué piensa o qué siente en un momento concreto (Stuss, Gallup, \& Alexander, 2001).

El ejemplo clásico de esta habilidad es el Test de los Smarties. En esta prueba, se le enseña un paquete de caramelos a un niño y se le pregunta qué hay en él. Lógicamente, el niño responde "caramelos». El examinador abre el paquete y muestra al niño el contenido: un lapicero. Posteriormente, se le dice al niño que un amigo va a entrar en la habitación y que se le va a preguntar qué hay en el paquete. El primer niño debe intentar responder qué va a decir el segundo niño. La respuesta correcta sería «caramelos» puesto el segundo niño no sabe que hay un lapicero. La respuesta errónea sería «un lápiz» puesto que el primer niño no tiene en cuenta lo que sabe o piensa el segundo.

A pesar del debate entre las distintas hipótesis sobre cómo funciona la ToM y cuáles son sus subprocesos (Sabbagh, 2004), resulta plausible considerar que la capacidad de saber qué piensa, siente o quiere el otro es importante para relacionarnos socialmente. De hecho, varios estudios relacionan un bajo rendimiento en tareas de ToM con conductas socialmente inapropiadas (Lough \& Hodges, 2002), así como con lesiones ventromediales $\mathrm{u}$ orbitofrontales (Shamay-Tsoory et al., 2005).

Otro de los aspectos incluidos en la red de cognición social, es la capacidad de reconocer la emoción en otro a través de la expresión mostrada en el rostro o en la voz. Hornak y colaboradores realizaron un estudio (Hornak, Rolls, \& Wade, 1996) en el que pusieron a prueba la 
capacidad de personas con lesiones ventromediales de reconocer la emoción en una persona a través de expresiones en el rostro y en la voz. Los resultados sugirieron que los pacientes con conductas socialmente inapropiadas tenían más dificultades que otros pacientes para reconocer expresiones emocionales. No resulta descabellado aceptar que reconocer emociones puede ser una de las claves para saber qué esta sintiendo o pensando el otro. Así, este podría ser un paso previo a la capacidad de mentalizar de forma exitosa.

La red de cognición social incluye, a niveles más básicos, procesos como percepción de movimiento de seres biológicos (frente a seres mecánicos), la inferencia de intenciones de un agente en movimiento, la capacidad de imitación, etc. (Bird, Castelli, Malik, Frith, \& Husain, 2004), aunque aún no se han realizado estudios que relacionen estas capacidades con aspectos más clínicos, como los trastornos de conducta en daño cerebral.

\section{La apatía:}

Equivalente al síndrome apático, la apatía se presenta como una de las patologías menos estudiadas desde la neuropsicología cognitiva. La mayor parte de los estudios realizados se centran en aspectos clínicos de este trastorno. Una de las contribuciones más importantes de estos estudios es la de señalar la diferencia existente entre apatía como síndrome y apatía como síntoma (Marin, 1991). Existen patologías (trastornos del estado de ánimo, alteraciones de la conciencia, etc,) que presentan apatía como un síntoma más, y deben diferenciarse del síndrome apático, cuya causa no es uno de los trastornos mencionados, sino que es una entidad clínica en sí misma (van Reekum, Stuss, \& Ostrander, 2005).

La apatía se manifiesta en forma de disminución de la actividad que el paciente inicia por sí mismo (acción autoiniciada) por falta de motivación, no atribuible a un trastorno del intelecto, emoción o nivel de conciencia. Se evidencia por tres aspectos fundamentales, la disminución de la conducta, de la cognición y de la emoción (ver tabla 1).

Por ejemplo, cuando se le confronta con pérdidas personales, problemas de salud o económicos, el paciente apático se muestra emocionalmente indiferente, plácido, inapropiadamente eufórico, con afecto superficial o plano. Del mismo modo, los sucesos favorables provocan respuestas emocionales atenuadas.

En cuanto a los modelos explicativos de la apatía, apenas existen propuestas publicadas. Una de las más interesantes es la que proponen Stuss y colaboradores (Alexander \& Stuss, 2000), partiendo de la base de que la apatía es la falta de acción autoiniciada. Entonces, una función afectada (p. ej. movimientos oculomoto-

TABLA 1. Características clínicas de la apatía

\begin{tabular}{|c|c|c|}
\hline $\begin{array}{l}\text { Características de la disminución } \\
\text { de la conducta }\end{array}$ & $\begin{array}{c}\text { Características de la disminución } \\
\text { de la cognición }\end{array}$ & $\begin{array}{l}\text { Características de la disminución } \\
\text { de la emoción }\end{array}$ \\
\hline $\begin{array}{l}\text { - falta de productividad, } \\
\text { > falta de esfuerzo, } \\
\text { - falta de tiempo invertido e ini- } \\
\text { ciativa, } \\
\text { > aumento de perseveraciones, } \\
\text { > conductas de sumisión o depen- } \\
\text { dencia, } \\
\text { > disminución de la socialización } \\
\text { y de actividades de ocio. }\end{array}$ & $\begin{array}{l}\text { falta de interés tanto a nivel ge- } \\
\text { neral como en el aprendizaje de } \\
\text { nuevos conocimientos o expe- } \\
\text { riencias, } \\
\text { > despreocupación por uno mismo, } \\
\text { la propia salud y los propios } \\
\text { problemas, } \\
\text { disminución del valor atribuido } \\
\text { a la socialización, recreación, } \\
\text { productividad, iniciativa, perse- } \\
\text { verancia curiosidad. }\end{array}$ & $\begin{array}{l}>\text { afectividad invariable, } \\
\text { falta de sensibilidad emocional } \\
\text { hacia sucesos positivos o negati- } \\
\text { vos, } \\
\text { > aplanamiento afectivo, } \\
\text { > ausencia de excitación o intensi- } \\
\text { dad emocional. }\end{array}$ \\
\hline
\end{tabular}


res) provocaría una apatía hacia los estímulos que esa función procesa (p. ej. estímulos visuales). Desde este punto de vista, toda dificultad en reaccionar ante estímulos sería un tipo de apatía, diferente según el circuito afectado. En otro ejemplo, una dificultad de planificación provocaría una apatía en el sentido de falta de iniciación de conductas con un objetivo. Esto se pone de manifiesto en el síndrome disejecutivo, en cuyas descripciones se incluye una cierta apatía (Duffy \& Campbell, 1994).

Otros conceptos como mutismo acinético y abulia son patologías con las que comparte características clínicas y mecanismos fisiológicos, aunque se diferenciaría de aquellas por la apatía de un trastorno más leve (Marin \& Wilkosz, 2005). Las tres patologías descritas se suelen agrupar bajo el epígrafe «trastornos de motivación disminuida».

\section{Evaluación de los trastornos de conducta}

La evaluación de las patologías incluidas en el constructo trastorno orgánico de personali- dad, presenta una serie de dificultades metodológicas que no suele ser aplicable a otros déficit tras una lesión cerebral, tal y como señalan Pelegrín y colaboradores (Pelegrín, Gómez, Muñoz-Céspedes, Fernández, \& Tirapu, 2001) (ver tabla 2).

Además de estos problemas, se añade el hecho de que la conceptualización de las alteraciones de conducta se encuentra en desarrollo, algo que influye de forma determinante para alcanzar un nivel óptimo de validez del constructo a evaluar. Estas dificultades son, al menos en parte, las responsables del notable retraso existente en los avances realizados en la evaluación de los subtipos de TOP respecto a la de funciones cognitivas como la memoria o el lenguaje.

A pesar de lo anterior, existen instrumentos disponibles para valorar estas patologías. Fundamentalmente pueden dividirse en dos grandes grupos: las escalas y los test.

Las escalas de evaluación han sido el instrumento tradicional para evaluar los trastornos de conducta. Suele tratarse de herramientas provenientes del ámbito neuropsiquiátrico y va-

\section{TABLA 2. Dificultades metodológicas existentes en la evaluación del paciente con trastorno orgánico de personalidad}

- Inestabilidad de los síntomas, especialmente en los seis primeros meses tras la lesión.

- Necesidad de evaluar la personalidad premórbida del paciente.

- La relativa ausencia de instrumentos estándar que evalúen esta patología.

- El probable déficit en la capacidad de introspección del paciente.

- La necesidad de controlar factores de posible confusión, como trastornos físicos, déficit cognitivos o situaciones sociales adversas.

- La necesidad de diferenciar rasgos y estados.

- La necesidad de diferenciar rasgos y situaciones.

- La posible presencia de pacientes o familiares poco colaboradores o inestables emocionalmente.

- La probable escasa fiabilidad de los datos proporcionados por los familiares debido a (1) la negación de síntomas por parte de sus parientes, (2) la modificación en la atribución al considerar la lesión como causa de conductas debidas a otros factores y (3) la exageración de síntomas al descender su nivel de tolerancia hacia el paciente o por la existencia de ganancia secundaria de tipo económico.

- Los posibles falsos positivos o falsos negativos en relación a las características culturales, normas sociales y ética del informador.

- Las diferencias en la interpretación de la información obtenida por parte de varios clínicos (fiabilidad interevaluadores) o en diferentes momentos de la evolución (fiabilidad test-retest). 
loran fundamentalmente conductas, sobre la base de su presencia, frecuencia o intensidad.

Existen escalas generales de trastornos de conducta, como la Neurobehavioural Rating Scale (Goldstein \& Levin, 1987), de la que existe una versión española (Pelegrin \& Tirapu, 1995), o la Frontal Systems Behavior Scale (FrSBe), la cual merece ser comentada en mayor detalle.

La FrSBe es una actualización de la Frontal Lobe Personality Scale (FLOPS) y valora los trastornos conductuales asociados a la lesión de los circuitos cerebrales fronto-subcorticales (Stout, Ready, Grace, Malloy, \& Paulsen, 2003). Consta de 46 ítems de cinco opciones de respuesta a elegir en función de la frecuencia de la conducta (desde Casi nunca hasta Casi siempre). Existe una versión a cumplimentar por el paciente y otra por un familiar. Ambos deben responder a todos los ítems acerca de la conducta pre- y postmórbida. El contenido de los ítems puede dividirse en tres grupos (apatía, desinhibición y disfunción ejecutiva), división basada en la propuesta de los tres síndromes prefrontales (Duffy \& Campbell, 1994) expuesta previamente.

Por otra parte, existen escalas específicas, como la Apathy Evaluation Scale (Marin, Biedrzycki, \& Firinciogullari, 1991), diseñada con el objetivo de valorar apatía. Este instrumento consta de dieciocho ítems (ver tabla 3 ), que se puntúan sobre la base de frecuencia de ocurrencia de la conducta descrita. Existen cuatro opciones de respuesta (nunca, casi nunca, a veces, siempre) y tiene tres formas de aplicación en función de quién la cumplimenta (del clínico, del familiar y del paciente). Las propiedades psicométricas han sido descritas en (Marin et al., 1991).

Por otra parte, los test neuropsicológicos existentes provienen del ámbito de la neuropsi-

TABLA 3. Items de la Apathy Evaluation Scale

\begin{tabular}{|l|}
\hline 1. Se interesa por las cosas. \\
\hline 2. Hace cosas durante el día. \\
\hline 3. Empezar a hacer algo por su cuenta es importante para él/ella. \\
\hline 4. Se interesa por tener nuevas experiencias. \\
\hline 5. Se interesa por aprender cosas nuevas. \\
\hline 6. Se esfuerza poco en las cosas. \\
\hline 7. Vive la vida con intensidad. \\
\hline 8. Observar terminada una labor es importante para él/ella. \\
\hline 9. Emplea el tiempo haciendo cosas que le interesan. \\
\hline 10. Alguien tiene que decirle lo que hacer cada día. \\
\hline 11. Está menos preocupado por sus problemas de lo que debería. \\
\hline 12. Tiene amigos. \\
\hline 13. Reunirse con los amigos es importante para él/ella. \\
\hline 14. Cuando sucede algo bueno, se entusiasma. \\
\hline 15. Tiene una comprensión acertada de sus problemas. \\
\hline 16. Estar haciendo cosas durante el día es importante para él/ella. \\
\hline 17. Tiene iniciativa. \\
\hline 18. Tiene motivación. \\
\hline
\end{tabular}


cología cognitiva, se encuentran en fases tempranas de estudio y están enfocados para evaluar procesos subyacentes a las conductas. Fundamentalmente existen instrumentos para valorar procesos subyacentes a la desinhibición conductual y aún no se han desarrollado tareas para la apatía. Las tareas más representativas son:

- Gambling Task (Bechara, Damasio, Damasio, \& Anderson, 1994):

Esta tarea fue diseñada con el objetivo de estudiar la hipótesis de los marcadores somáticos (Damasio, 1996). En ella, se le indica al paciente que se encuentra ante un juego en el que su objetivo es conseguir la mayor cantidad de dinero posible. Se le presentan cuatro barajas llamadas A, B, C y D, y se le informa de que cada carta que elija le aportará una cantidad variable de dinero. Algunas de las cartas, además, le restarán ganancias. Se trata de una tarea que finaliza cuando el paciente coge 100 cartas. Las barajas A y B, aportan cantidades elevadas de dinero, por lo que resultan atractivas. Sin embargo, las cifras que restan son muy elevadas. Las barajas C y D, por el contrario, otorgan menores cantidades económicas, aunque sus penalizaciones son menores. Por lo tanto, la elección mayoritaria de cartas de las barajas A y B resulta en una ejecución deficiente, mientras que seleccionar preferentemente las barajas $\mathrm{C}$ y D permite alcanzar el objetivo de la tarea.

- Tareas de reconocimiento de expresiones emocionales

Este tipo de tareas consisten en presentar al sujeto imágenes de varias personas expresando una emoción con el rostro. Su labor consiste en seleccionar qué emoción está expresando la persona de la imagen, de entre varias opciones de respuesta, que suelen incluir la tristeza, la ira, la alegría, el asco, la tristeza y la sorpresa, por ser las universalmente igual expresadas por el ser humano (FernándezAbascal \& Chóliz-Montañés, 2001).

Según recientes trabajos (Hornak et al., 2003) los pacientes desinhibidos reconocen peor las emociones que otros grupos experimentales (controles y pacientes no desinhibidos). También se ha estudiado esta cuestión con una tarea de reconocimiento de emoción en la voz, con resultados similares.

\section{- Tareas de teoría de la mente}

Existen varias tareas que valoran la capacidad denominada "teoría de la mente». Lo esencial de estas tareas es que todas requieren que el sujeto al que se le aplican sea capaz de inferir estados mentales en el otro. Algunas de ellas son:

- En el Test Avanzado de ToM, el evaluador lee una historia, después realizará al sujeto una serie de preguntas que éste deberá responder. Para responder de forma correcta, es necesario que el sujeto sea capaz de realizar inferencias acerca del estado mental, pensamientos, sentimientos, intenciones, etc. del personaje. Se anotan las respuestas literales del sujeto y se analizan los términos que usa para explicar la conducta del personaje (pensaba, dar por hecho, sabía, esperaba...). Según las respuestas dadas, se puede determinar si el sujeto es capaz de mentalizar o no (Fletcher et al., 1995).

- El Test de Incumplimiento de Normas Sociales consta de una serie de historias cortas que describen un una situación social. Cada una de las historias tiene tres finales diferentes que se corresponden con diferentes situaciones (situación embarazosa, situación inapropiada y situación normal). La situación embarazosa, es aquella en la que el personaje viola una norma social inintencionadamente, en la situación inapropiada, el personaje viola una norma social de forma intencionada, en el tercero de los casos (situación normal) se describe una situación normal. El evaluador lee al sujeto la historia con los tres finales diferentes, el sujeto tiene que decidir a qué tipo de situación pertenece cada final (Berthoz, Armony, Blair, \& Dolan, 2002).

- El Faux Pas Test consta de varias historias acerca de diferentes situaciones sociales. En la mitad de ellas, un personaje dice algo inapropiado. Al sujeto se le lee la historia y debe identificar al sujeto que ha dicho lo inapropiado y entonces debe explicar por qué (Baron-Cohen et al., 1999). 
- En el Test de Animaciones. Se presenta al sujeto una animación en la unos objetos realizan movimientos. El sujeto debe atribuir estados mentales o intenciones a dichos objetos. Algunas de las animaciones describen movimientos de pelea, al azar, que uno trate de expulsar a otro de una figura geométrica, etc. En todos los casos, se anotan las repuestas literales de los sujetos (Castelli, Frith, Happe, \& Frith, 2002).

Diversos estudios han obtenido correlaciones entre la presencia de desinhibición conductual y una dificultad para realizar este tipo de tareas (Lough \& Hodges, 2002; Rowe, Bullock, Polkey, \& Morris, 2001; ShamayTsoory, Tomer, Berger, \& Aharon-Peretz, 2003). El marco conceptual desde el que se proponen estas tareas no es tan evidente como en la tarea del casino y en otras comentadas anteriormente, sino que está en proceso de desarrollo. Algunos autores como Sabbagh (2004) han comenzado a plantear hipótesis de funcionamiento de los procesos que permiten el funcionamiento de la teoría de la mente.

\section{- Tareas Go - No Go}

Este tipo de tareas no se diseñaron con el propósito de valorar específicamente desinhibición, aunque su ejecución deficitaria se ha relacionado con esta patología (Malloy, Bihrle, Duffy, \& Cimino, 1993). Estas tareas consisten en la presentación (normalmente en una pantalla de ordenador, pero también por parte del examinador) de una serie de estímulos (p. ej. letras) de uno en uno, ante los que el sujeto debe dar una respuesta motora (p. ej. apretar un botón). Además se le indica que ante un tipo determinado de estímulo no debe responder (p. ej. ante la letra A) y se contabilizan las veces que el sujeto responde por error a estos estímulos (falsos positivos) y las que no responde cuando sí debería hacerlo (omisiones). En los pacientes que presentan lesiones en la zona medial de los lóbulos frontales se observa un mayor número de falsos positivos, en comparación con otros grupos experimentales (Malloy et al., 1993; Spinella, 2004).

\section{Rehabilitación de los trastornos de conducta}

La intervención en los trastornos de conducta es uno de los principales objetivos que se establece en la práctica clínica. Su importancia es debida a que este tipo de alteraciones impacta sobre todas las áreas de la persona y su entorno. En primer lugar, supone el principal obstáculo en la reintegración laboral del paciente (Brooks, McKinlay, Symington, Beattie, \& Campsie, 1987; McKinlay, Brooks, Bond, Martinage, \& Marshall, 1981). Por ejemplo, un paciente con trastornos de memoria quizá no pueda reincorporarse a su puesto, pero sí puede trabajar en otro que no exija sus capacidades perdidas. En cambio, un paciente sin motivación, o sin capacidad de aceptar normas, no podrá desempeñar labor alguna.

En segundo lugar, la familia recibe un impacto emocional mayor que en el caso de trastornos cognitivos como agnosias, etc. Estos déficit son vividos como discapacidades que impiden al paciente desenvolverse como antes de la lesión, pero la persona sigue siendo ella misma. En cambio, la apatía y la desinhibición provocan que la familia tenga la vivencia de que el paciente es otra persona diferente (Pelegrín et al., 2001).

Tercero, la propia rehabilitación (neuropsicológica, aunque también física, logopédica, ocupacional, etc.) se ve afectada por la conducta del paciente. La falta de motivación y, por lo tanto, de implicación en la terapia, así como la falta de respeto a los terapeutas y a las normas del proceso rehabilitador son obstáculos muy importantes para el resultado exitoso de la intervención (van Reekum et al., 2005).

Existen tres conceptos clave para entender la filosofía de la rehabilitación neuropsicológica (Muñoz Cespedes \& Tirapu, 2001):

a) La restauración: la idea fundamental es que la función afectada puede recuperarse mediante el ejercicio repetido. Este enfoque ha demostrado cierta validez en la rehabilitación de alteraciones de la atención (p. ej. el tiempo que un paciente puede atender a una tarea aumenta notablemente con la práctica) y de funcio- 
nes motoras, pero no en trastornos de la memoria.

b) La sustitución: se trata de que el paciente pueda hacer la misma tarea que hacía antes de la lesión utilizando otras funciones conservadas. Por ejemplo al paciente con heminegligencia izquierda se le puede entrenar a girar la cabeza hacia la izquierda cada vez que cruza una puerta, de modo que no se choque con el marco. En este ejemplo, una función perdida (la atención al hemicampo izquierdo) es sustituida por otra conservada (el recuerdo y la conciencia de cuál es el problema y de cuál es la solución).

c) La compensación: consiste en la búsqueda de estrategias alternativas o ayudas externas que reduzcan la implicación de las funciones afectadas (p. ej. una agenda para un paciente que olvida las citas). El éxito de esta aproximación radica en la correcta elección de la ayuda externa y en el desarrollo del aprendizaje necesario para su uso.

En general, a medida que transcurre el tiempo desde la lesión se va optando por métodos de tipo compensatorios y menos por los restauradores. En el caso de las alteraciones de conducta, se puede entrenar a los pacientes a compensar sus conductas deficitarias. Por ejemplo, re-entrenar la conducta apropiada según cada situación, de modo automático, sin depender del correcto procesamiento de la situación por parte del paciente, o independientemente de que tenga o no motivación para hacerlo. Sin embargo, en la práctica clínica este tipo de intervenciones no suelen ser exitosas, y se tiende a enfoques más específicos.

En este sentido, existen cuatro enfoques en la rehabilitación neuropsicológica de las alteraciones de conducta (Sohlberg \& Mateer, 2001):

1) Las terapias de control del entorno: Consiste en modificar las influencias que puedan alterar la conducta del paciente, tales como visitas, ruidos, iluminación, etc.

2) Las estrategias de comunicación: Se centra en mejorar las habilidades sociales del paciente, así como los estilos comunicativos de sus familiares o cuidadores.
3) La terapia de conducta: Trata de identificar y registrar los estímulos que provocan conductas no deseadas, así como las propias conductas. Se implementa sobre el registro una serie de refuerzos y castigos para cada conducta, para que los comportamientos deseados aumenten de frecuencia y desaparezcan los no deseados. Las conductas tienden a repetirse si se refuerzan (con atención, halagos, etc.) y a desaparecer si se castigan (ignorándolos, etc.). Los refuerzos que más provocan el mantenimiento de una conducta deseada son los que ocurren con una frecuencia alta, pero no siempre.

4) Las terapias de autocontrol: Su objetivo es que el paciente sea consciente de qué conductas debe evitar y cuáles repetir, de qué conducta es apropiada en un entorno y cuál no, y de qué puede hacer para evitar una conducta determinada. Incluye aprender a reconocer las diferentes situaciones sociales, a saber cuáles son las conductas apropiadas y a hacer respiraciones y relajación muscular rápida para auto-controlarse.

Tradicionalmente, las intervenciones que se realizan en este grupo de patologías son de terapia de conducta y de tipo farmacológico. Estas estrategias han demostrado utilidad en la agresividad y algo menos en desinhibición, aunque en el caso de la apatía su efectividad resulta limitada.

Además de estas perspectivas, los nuevos enfoques que tratan de explicar mecanismos subyacentes a las patologías, como la ToM, podrían ser útiles para sugerir nuevas estrategias de intervención. A medida que se avance en el conocimiento de cuáles son los procesos implicados en estas patologías, será posible igualmente avanzar hacia técnicas terapéuticas más certeras y específicas para las alteraciones de conducta.

Por otro lado, la intervención en alteraciones de conducta debe incluir obligatoriamente la psicoeducación a los familiares del paciente. Los objetivos de la psicoeducación son ayudar al familiar a entender la naturaleza de las secuelas y evitar atribuciones morales a esa conducta («se ha vuelto un egoísta, un desagradecido»), situar las expectativas en rangos pronósticos 
realistas e incorporar al cuidador y a otros miembros de la familia a la reflexión sobre cómo mejorar la calidad de vida.

\section{Referencias}

Alexander, M. P., \& Stuss, D. T. (2000). Disorders of frontal lobe functioning. Semin Neurol, 20(4), 427-437.

Baron-Cohen, S., Ring, H. A., Wheelwright, S., Bullmore, E. T., Brammer, M. J., Simmons, A., \& Williams, S. C. (1999). Social intelligence in the normal and autistic brain: an fMRI study. Eur $J$ Neurosci, 11(6), 1891-1898.

Bechara, A., Damasio, A. R., Damasio, H., \& Anderson, S. W. (1994). Insensitivity to future consequences following damage to human prefrontal cortex. Cognition, 50(1-3), 7-15.

Benton, A. L. (1991). The prefrontal region: its early history. In H. S. Levin \& H. M. Eisenberg \& A. L. Benton (Eds.), Frontal lobe function and dysfunction (pp. 3-29). Nueva York: Oxford University Press.

Bernhardt, M., \& Borchardt, M. (1909). Zur Klinik der Stirnhirntumoren nebst Bemerkungen über Hirnpunktion. Berliner Klinische Wochenschrift, 46, 1341-1347.

Berthoz, S., Armony, J. L., Blair, R. J., \& Dolan, R. J. (2002). An fMRI study of intentional and unintentional (embarrassing) violations of social norms. Brain, 125(Pt 8), 1696-1708.

Bird, C. M., Castelli, F., Malik, O., Frith, U., \& Husain, M. (2004). The impact of extensive medial frontal lobe damage on 'Theory of Mind' and cognition. Brain.

Brooks, D. N., McKinlay, W., Symington, C., Beattie, A., \& Campsie, L. (1987). Return to work within seven years if severe head injury. Brain Injury, 1, 5-19.

Castelli, F., Frith, C., Happe, F., \& Frith, U. (2002). Autism, Asperger syndrome and brain mechanisms for the attribution of mental states to animated shapes. Brain, 125(Pt 8), 1839-1849.

Cummings, J. L. (1998). Frontal-subcortical circuits and human behavior. J Psychosom Res, 44(6), 627-628.

Damasio, A. R. (1996). El error de Descartes. Barcelona: Crítica.
Duffy, J. D., \& Campbell, J. J., 3rd. (1994). The regional prefrontal syndromes: a theoretical and clinical overview. J Neuropsychiatry Clin Neurosci, 6(4), 379-387.

Fernández-Abascal, E. G., \& Chóliz-Montañés, M. (2001). Expresión facial de la emoción. Madrid: UNED Ediciones.

Feuchtwanger, E. (1923). Die Funktionen des Stirnhirns. Berlin: Springer.

Fletcher, P. C., Happe, F., Frith, U., Baker, S. C., Dolan, R. J., Frackowiak, R. S., \& Frith, C. D. (1995). Other minds in the brain: a functional imaging study of "theory of mind" in story comprehension. Cognition, 57(2), 109-128.

Goldstein, F. C., \& Levin, H. S. (1987). Disorders of reasoning and problem-solving ability, Neuropsychological rehabilitation (?) (pp. 327-378).

Halligan, P. W., \& David, A. S. (2001). Cognitive neuropsychiatry: towards a scientific psychopathology. Nat Rev Neurosci, 2(3), 209-215.

Harlow, J. M. (1848). Passage of an iron bar through the head. Boston Med Surg J, 39, 389-393.

Harlow, J. M. (1868). Recovery from the passage of an iron bar through the head. Publ Mass Med Soc, 2, 327-347.

Hebb, D. O. (1945). Man's frontal lobes: a critical review. Archives of Neurology and Psychiatry, 54, 1024.

Hornak, J., Bramham, J., Rolls, E. T., Morris, R. G., O'Doherty, J., Bullock, P. R., \& Polkey, C. E. (2003). Changes in emotion after circumscribed surgical lesions of the orbitofrontal and cingulate cortices. Brain, 126(Pt 7), 1691-1712.

Hornak, J., Rolls, E. T., \& Wade, D. (1996). Face and voice expression identification in patients with emotional and behavioural changes following ventral frontal lobe damage. Neuropsychologia, 34(4), 247-261.

Jastrowitz, M. (1888). Beiträge zur Lokalisation im Grosshirn und über deren praktische Verwerthung. Deutsche Medizinische Zeitschrift, 14, 8183, 108-112, 125-128, 151-153, 172-175, 188-192, 209-211.

Lough, S., \& Hodges, J. R. (2002). Measuring and modifying abnormal social cognition in frontal variant frontotemporal dementia. $J$ Psychosom Res, 53(2), 639-646. 
Malloy, P. F., Bihrle, A., Duffy, J., \& Cimino, C. (1993). The orbitomedial frontal syndrome. Archives of Clinical Neuropsychology, 8(3), 185-201.

Marin, R. S. (1991). Apathy: a neuropsychiatric syndrome. J Neuropsychiatry Clin Neurosci, 3(3), 243254.

Marin, R. S., Biedrzycki, R. C., \& Firinciogullari, S. (1991). Reliability and validity of the Apathy Evaluation Scale. Psychiatry Res, 38(2), 143-162.

Marin, R. S., \& Wilkosz, P. A. (2005). Disorders of diminished motivation. J Head Trauma Rehabil, 20(4), 377-388.

McKinlay, W., Brooks, D. N., Bond, M. R., Martinage, D. P., \& Marshall, M. M. (1981). The short-term outcome of severe blunt head injury as reported by relatives of injured persons. $J$ Neurol Neurosurg Psychiatry, 44, 527-533.

Moreno Gea, P., \& Blanco Sánchez, M. C. (1997). Clasificación y epidemiología de los traumatismos craneoencefálicos. In C. Pelegrín \& J. M. Muñoz Céspedes \& J. I. Quemada (Eds.), Neuropsiquiatría del daño cerebral traumático (pp. 11-33). Barcelona: Prous Science.

Muñoz Cespedes, J. M., \& Tirapu, J. (2001). Rehabilitación neuropsicológica. Madrid: Síntesis.

Nigg, J. T. (2000). On inhibition/disinhibition in developmental psychopathology: views from cognitive and personality psychology and a working inhibition taxonomy. Psychol Bull, 126(2), 220-246.

Oppenheim, H. (1890). Zur Pathologie der Gerhirngeschwulste. Archiv für Psychiatrie, 21, 560-578.

Pelegrín, C., Gómez, R., Muñoz-Céspedes, J. M., Fernández, S., \& Tirapu, J. (2001). Consideraciones nosologicas del cambio de personalidad postraumatico. Rev Neurol, 32(7), 681-687.

Pelegrin, C., \& Tirapu, J. (1995). Neuropsiquiatría del daño prefrontal traumático. Psiquiatría, 7(6), 11-21.

Quensel, F. (1914). Stirnhirnverletzung mit Charakterveränderung. Muenchener Medizinischer Wochenschrift, 6, 1761-1763.

Rowe, A. D., Bullock, P. R., Polkey, C. E., \& Morris, R. G. (2001). "Theory of mind" impairments and their relationship to executive functioning following frontal lobe excisions. Brain, 124(Pt 3), 600616.

Sabbagh, M. A. (2004). Understanding orbitofrontal contributions to theory-of-mind reasoning: implications for autism. Brain Cogn, 55(1), 209-219.
Sánchez-Cubillo, I., Muñoz-Céspedes, J. M., \& Quemada, J. I. (2004). ¿Qué procesos cognitivos están implicados en el trastorno orgánico de personalidad? Anales de Psicología, 20(2), 273-287.

Sánchez-Cubillo, I., Quemada, J. I., Muñoz Céspedes, J. M., Furundarena, A., \& García-Valcarce, M. (2003). Valoración neuropsicológica de los aspectos atencionales del trastorno orgánico de personalidad desinhibido. Paper presented at the RECA-4, Valencia.

Schuster, P. (1902). Psychische Störungen bei Hirntumoren. Stuttgart: Enke.

Shamay-Tsoory, S. G., Lester, H., Chisin, R., Israel, O., Bar-Shalom, R., Peretz, A., Tomer, R., Tsitrinbaum, Z., \& Aharon-Peretz, J. (2005). The neural correlates of understanding the other's distress: a positron emission tomography investigation of accurate empathy. Neuroimage, 27(2), 468-472.

Shamay-Tsoory, S. G., Tomer, R., Berger, B. D., \& Aharon-Peretz, J. (2003). Characterization of empathy deficits following prefrontal brain damage: the role of the right ventromedial prefrontal cortex. J Cogn Neurosci, 15(3), 324-337.

Singer, T., Seymour, B., O'Doherty, J., Kaube, H., Dolan, R. J., \& Frith, C. D. (2004). Empathy for pain involves the affective but not sensory components of pain. Science, 303(5661), 1157-1162.

Sohlberg, M. M., \& Mateer, C. A. (2001). Cognitive rehabilitation: an integrative neuropsychological approach (1. ed.). New York: The Guilford Press.

Spinella, M. (2004). Neurobehavioral correlates of impulsivity: evidence of prefrontal involvement. Int J Neurosci, 114(1), 95-104.

Stout, J. C., Ready, R. E., Grace, J., Malloy, P. F., \& Paulsen, J. S. (2003). Factor analysis of the frontal systems behavior scale (FrSBe). Assessment, 10(1), 79-85.

Stuss, D. T., Gallup, G. G., Jr., \& Alexander, M. P. (2001). The frontal lobes are necessary for 'theory of mind'. Brain, 124(Pt 2), 279-286.

van Reekum, R., Stuss, D. T., \& Ostrander, L. (2005). Apathy: why care? J Neuropsychiatry Clin Neurosci, 17(1), 7-19.

Vincent, C. (1936). Neurochirurgische Betrachtungen über die Funktionen des Frontallappens. Deutsche Medizinische Wochenschrift, 62, 41-45.

Welt, L. (1888). Über Charakterveränderungen des Menschen infolge von Läsionen des Stirnhirns. Deutsche Archiv für Klinische Medizin, 42, 339-390. 